\title{
Understanding and Reducing Photothermal Forces for the Fabrication of Au Nanoparticle Dimers by Optical Printing
}

\author{
Julian Gargiulo, ${ }^{\dagger}$ Thomas Brick, ${ }^{\ddagger}$ Ianina L. Violi, ${ }^{\dagger}$ Facundo C. Herrera, " Toshihiko Shibanuma, ${ }^{\ddagger}$ \\ Pablo Albella, ${ }^{\ddagger} \S$ Félix G. Requejo, ${ }^{\|}$Emiliano Cortés, ${ }^{\ddagger}$ Stefan A. Maier, ${ }^{\ddagger}$ and Fernando D. Stefani* ${ }^{* \dagger, \perp}$ \\ ${ }^{\dagger}$ Centro de Investigaciones en Bionanociencias (CIBION), Consejo Nacional de Investigaciones Científicas y Técnicas (CONICET), \\ Godoy Cruz 2390, C1425FQD Ciudad de Buenos Aires, Argentina \\ ${ }^{\ddagger}$ The Blackett Laboratory, Department of Physics, Imperial College London, London SW7 2AZ, United Kingdom \\ ${ }^{\S}$ University Institute for Intelligent Systems and Numerical Applications in Engineering (SIANI), University of Las Palmas de Gran \\ Canaria, 35017, Las Palmas de Gran Canaria, Spain \\ "Instituto de Investigaciones Fisicoquímicas Teóricas y Aplicadas (INIFTA, CONICET), Departamento de Química, Facultad de \\ Ciencias Exactas, Universidad Nacional de La Plata, Diagonal 113 y 64, 1900 La Plata, Argentina \\ ${ }^{\perp}$ Departamento de Física, Facultad de Ciencias Exactas y Naturales, Universidad de Buenos Aires, Güiraldes 2620, C1428EAH \\ Ciudad de Buenos Aires, Argentina
}

Supporting Information

ABSTRACT: Optical printing holds great potential to enable the use of the vast variety of colloidal nanoparticles (NPs) in nano- and microdevices and circuits. By means of optical forces, it enables the direct assembly of NPs, one by one, onto specific positions of solid surfaces with great flexibility of pattern design and no need of previous surface patterning. However, for unclear causes it was not possible to print identical NPs closer to each other than $300 \mathrm{~nm}$. Here, we show that the repulsion restricting the optical printing of close by NPs arises from light absorption by the printed NPs and subsequent local heating. By optimizing heat dissipation, it is possible to reduce the minimum separation between NPs. Using a reduced graphene oxide layer on a sapphire substrate, we demonstrate for the first time the optical printing of $\mathrm{Au}-\mathrm{Au}$ NP dimers. Modeling the experiments considering optical, thermophoretic, and thermo-osmotic forces we obtain a detailed understanding and a clear pathway for the optical printing fabrication of complex nano structures and circuits based on connected colloidal NPs.

KEYWORDS: Plasmonics, optical forces, thermo-osmosis, thermophoresis, colloidal patterning, reduced graphene oxide, graphene
C olloidal synthesis methods permit the high-throughput and low-cost fabrication of colloidal nanoparticles (NPs) with precise control of composition, size, and shape. ${ }^{1-4}$ These nanomaterials have a number of advantages in comparison to their counterparts produced by top-down lithographic or etching methods, such as versatility of geometries, superior crystallinity, the ease of tailoring surface chemistry, ${ }^{5}$ and the potential for large-scale production. Current efforts in the field are devoted to control the incorporation of colloidal NPs in two-dimensional (2D) and three-dimensional (3D) assemblies on solid surfaces, ${ }^{6-9}$ so as to use their unique physical and (bio)chemical properties in functional devices such as optical nanoantennas $^{10,11}$ or highly sensitive SERS platforms. ${ }^{12,13}$ Among the various methods for decorating surfaces with colloidal NPs, optical printing stands out because it enables the fabrication of patterns of arbitrary design on plane substrates with no need of any previous steps of physical or chemical patterning. Optical printing uses highly focused laser beams to capture individual NPs from the colloidal suspension and fix them into predefined locations of a substrate with high accuracy and flexibility of pattern design. ${ }^{14-17}$ This technique is particularly suitable for highly polarizable NPs such as metallic NPs near their plasmon resonance, because large optical forces are produced with moderate laser powers. Furthermore, optical resonances of NPs allow the selective deposition of different kinds of NPs using lasers of different wavelengths. ${ }^{18-20}$ Arrays of individual NPs can be prepared also by simultaneous optical printing using spatially modulated light fields. ${ }^{15}$

One of the key challenges of optical printing is to improve its lateral resolution, that is, its ability to deposit two NPs at a controlled separation distance. Since its first implementations, ${ }^{14,21,22}$ it was found that the optical printing process was disturbed by the interaction of the laser beam with the already fixed NPs. For the optical printing of $\mathrm{Au}$ and $\mathrm{Ag}$ nanospheres or nanorods, the interparticle distances were restricted to the size of the focus waist, typically $300 \mathrm{~nm}$ for a Gaussian

Received: June 27, 2017

Revised: August 9, 2017

Published: August 14, 2017 
A

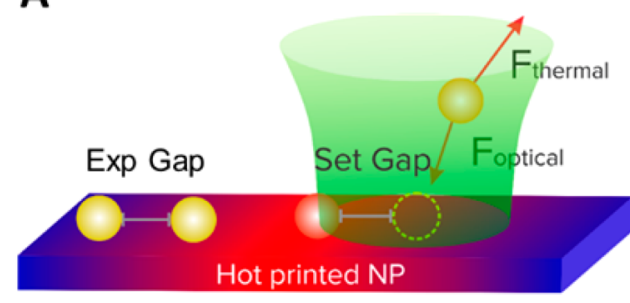

D

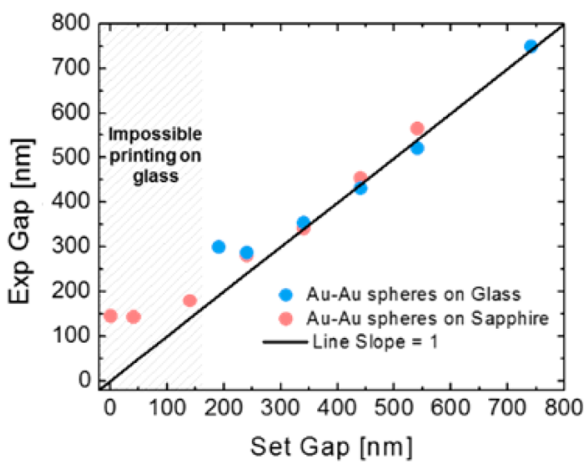

B

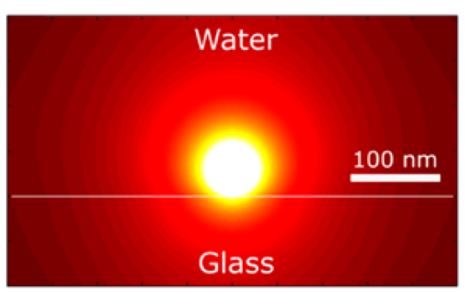

E

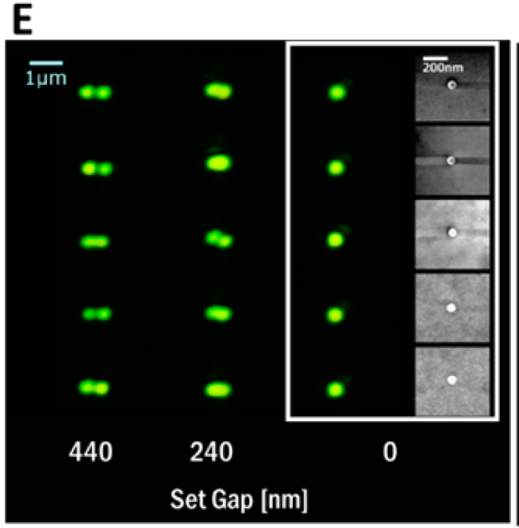

C

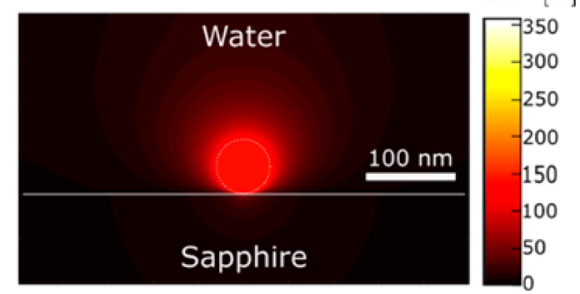

$F$

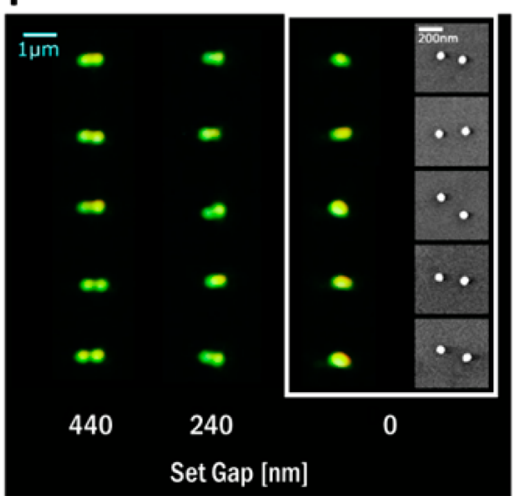

Figure 1. (A) Schematic of the $\mathrm{Au}-\mathrm{Au}$ dimers printing experiment and its relevant parameters and forces. As the laser beam illuminates the NPs on the surface, the local temperature increases. (B) Calculated temperature increase for a $60 \mathrm{~nm}$ Au NP on a glass substrate illuminated with a Gaussian beam with a waist of $266 \mathrm{~nm}$, wavelength of $532 \mathrm{~nm}$, and $P=1.2 \mathrm{~mW}$ and (C) on a sapphire substrate at $P=0.5 \mathrm{~mW}$, as in the experiments. (D) Measured experimental gap versus set gap for $\mathrm{Au}-\mathrm{Au} \mathrm{NP}$ dimers printed on glass and on sapphire substrates. Black line represents unperturbed printing. The shadowed region corresponds to set gaps where it was impossible to print a second NP on glass. (E,F) Dark-field images for $60 \mathrm{~nm}$ Au NP dimers printed at different set gaps on glass (E) and on sapphire (F). Insets show SEM images after printing with a set gap of $0 \mathrm{~nm}$.

diffraction-limited foci of visible wavelengths. ${ }^{16,23}$ In a recent report, ${ }^{24}$ we have shown that the repulsive interaction that prevents connecting NPs on a substrate could be avoided if the already printed NPs are transparent at the printing wavelength. Following this strategy, we were able to connect $\mathrm{Ag}$ and $\mathrm{Au}$ NPs with well-defined orientation. Still the exact mechanism behind the photoinduced repulsion remained unclear. In experiments where Ag NPs were first optically trapped and then printed, ${ }^{23}$ the limited interparticle distance could be clearly explained by optical binding. However, optical binding could not account for our observations and photothermal heating was then postulated to play an important role. Photothermal distortions have been reported on several other experiments of optical manipulation. ${ }^{25-29}$ Mechanistic discussions usually point toward convective currents induced by the hot NPs, ${ }^{30,31}$ although efforts to quantitatively explain the observed effects in terms of natural convection have systematically failed given their weak magnitude. ${ }^{32}$ Despite this limited understanding, nanoscale photothermal phenomena have found application in the manipulation of single ${ }^{33,34}$ and assemblies ${ }^{35}$ of NPs, molecules, ${ }^{36,37}$ as well as driving force for the motion of self-propelled Janus particles. ${ }^{38-40}$ Advances in the understanding of photothermal forces at the nanoscale are thus beneficial not only for optical printing but for an extended range of applications.

Here, we perform a systematic study of the lateral resolution of optical printing of Au NPs on substrates of different thermal conductivity. We show that using substrates with higher thermal conductivity, smaller interparticle distances can be achieved. Increasing the contact area between the metallic NP and the substrate is a complementary and effective way of increasing the heat-dissipation, as shown by printing spherical Au NPs next to Au nanodisks (NDs). The role of photothermal repulsion is clearly demonstrated. A complete modeling of the experiments considering optical, thermophoretic, and thermoosmotic forces provides detailed mechanistic insight. Finally, as a proof of concept, we show that by exploiting the high thermal conductivity of a reduced graphene oxide layer deposited on sapphire, it is possible to create dimers of connected Au NPs.

Results. Optical printing of $(63 \pm 3) \mathrm{nm}$ spherical Au NPs was performed using a CW laser with wavelength of $532 \mathrm{~nm}$, tuned to the localized surface plasmon resonance of the NPs. In a typical experiment, a diluted drop of colloidal citrate-capped negatively charged Au NPs is placed on top of the substrate to be patterned. Spontaneous adsorption of the NPs was avoided by functionalizing the substrate with a monolayer of polyelectrolyte rendering it with the same sign of charge as the NPs. The laser is focused to a Gaussian beam waist of (266 $\pm 3) \mathrm{nm}$ and the focal plane is set at the substrate, generating a small region where optical forces are stronger than the repulsion. NPs that diffuse into that region are pushed toward the beam center and the substrate, where they get fixed (printed) by the attractive van der Waals force. A minimum threshold laser power $P_{\text {th }}$ is required to overcome the electrostatic repulsion and print NPs. The process has a typical average positional accuracy of $50 \mathrm{~nm} .{ }^{14}$ Arrays of NPs are fabricated by automated subsequent printing of single NPs as described in our previous report. ${ }^{24}$ Further details on the experimental methods can also be found in SI.1.

Figure $1 \mathrm{~A}$ illustrates the temperature increase that takes place due to light absorption of a fixed NP when the printing beam is set to print a second NP nearby. In order to test the influence of this temperature increase on the lateral resolution of optical printing, Au NP dimers with different set gaps were printed on two substrates with considerably different thermal conductivities $(\kappa)$ : glass with $\kappa \sim 1 \mathrm{~W} / \mathrm{mK}$ and sapphire with $\kappa \sim 20 \mathrm{~W} /$ 
A

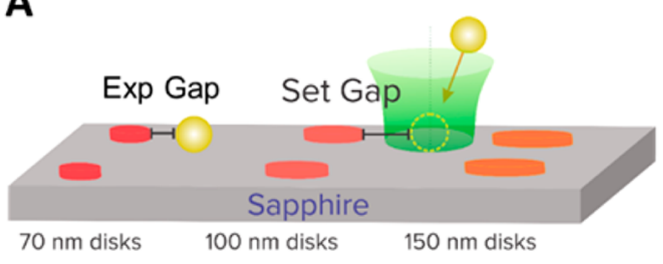

B

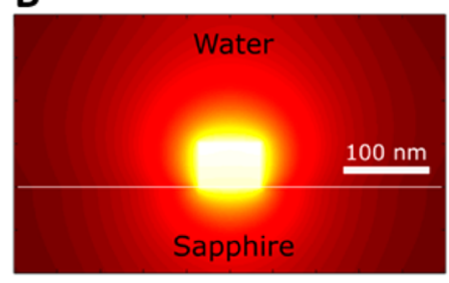

C

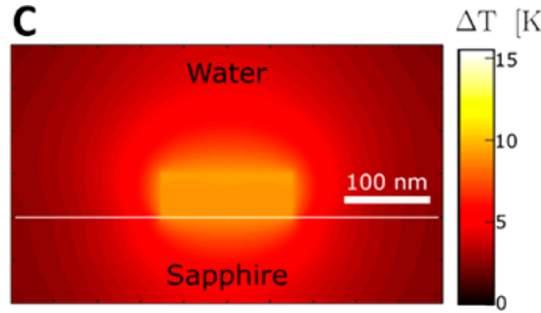

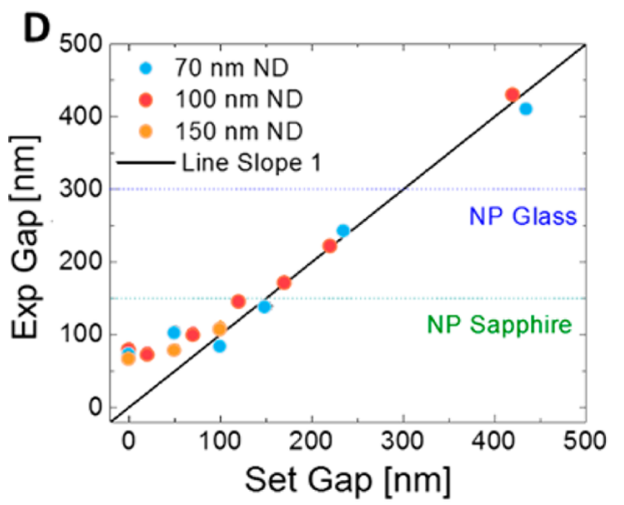

$\mathbf{E}$

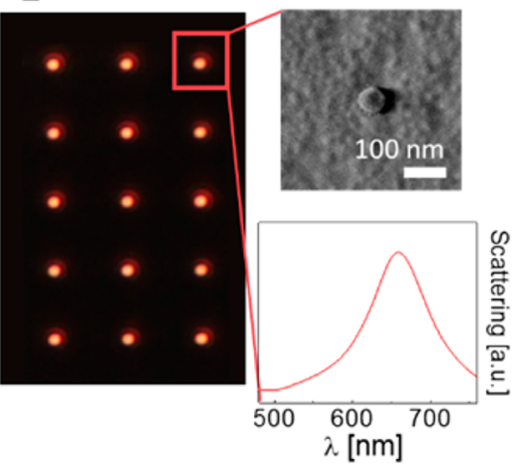

$\mathbf{F}$

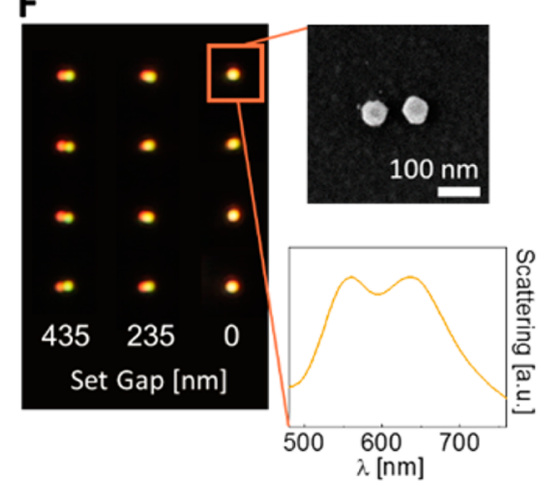

Figure 2. (A) Schematic of the AuND-AuNP dimers printing experiment. AuNDs had a height of $50 \mathrm{~nm}$ and diameters of 70, 100, and 150 nm. (B) Calculated temperature increase for a $70 \mathrm{~nm}$ Au ND and (C) $150 \mathrm{~nm}$ ND on a sapphire substrate illuminated with a beam of $P=0.5 \mathrm{~mW}$, as in the experiments. (D) Measured experimental gap versus set gap for AuND-AuNP dimers printed on sapphire. Black solid line represents unperturbed printing. Horizontal dashed lines represent the minimum achieved experimental gap for Au NPs dimers on glass and on sapphire. (E) Dark-field image of an array of $70 \mathrm{~nm}$ Au NDs, along with a representative FE-SEM image and scattering spectrum of a single ND. (F) Dark-field images of optically printed $\mathrm{Au}$ NPs next to $70 \mathrm{~nm}$ Au NDs for different set gaps (435, 235, and $0 \mathrm{~nm}$ ), along with a representative FE-SEM image and scattering spectrum of an obtained AuND-AuNP dimer for a set gap of $0 \mathrm{~nm}$.

$\mathrm{mK}$. The interparticle gaps are defined as the distance between the NPs from surface to surface. The resulting nanostructures, and in particular the interparticle separation gaps, were characterized by dark-field microscopy and field-emission scanning electron microscopy (FE-SEM; see SI.2 for details).

Figure 1B shows the calculated temperature map around a 60 $\mathrm{nm}$ Au NP on a glass substrate and illuminated with the center of a focused laser beam as the one used for printing with a power $P=1.2 \mathrm{~mW}$. The surrounding medium is water. Details about temperature calculations are provided in SI.7. Temperature increases of about $300 \mathrm{~K}$ can be achieved under these conditions. ${ }^{41}$ In agreement with previous reports, ${ }^{23,24}$ optical printing on glass substrates delivers precisely well-separated individual NPs to the set position but fails for interparticle gaps smaller than $300 \mathrm{~nm}$ (Figures 1D,E). For set gaps smaller than $150 \mathrm{~nm}$, optical printing of the second NP becomes impossible. Indeed, FE-SEM images confirmed that only a single NP is printed for those set gaps (example of set gap $0 \mathrm{~nm}$ in Figure 1E).

A different behavior was found on sapphire substrates. The first noticeable fact was that the threshold laser power for printing was roughly 2.4 times lower than on glass substrates. There are two plausible reasons for this. First, even though the chemical treatments of both surfaces were identical, it is possible that the sapphire substrates turned out with a lower charge density. Second, short-range attractive forces were predicted between a heat releasing particle and a thermally conductive substrate, as discussed in more detail in SI.3. ${ }^{42}$ Figure 1C shows the calculated temperature map around a 60 $\mathrm{nm} \mathrm{Au} \mathrm{NP}$ on a sapphire substrate (surrounding medium water) when placed at the center of the printing laser beam with power of $P=0.5 \mathrm{~mW}$. The combination of faster heat dissipation of sapphire and the lower laser power required for printing, leads to a 7-fold temperature reduction in comparison to the glass substrate. Using sapphire substrates, the precision of optical printing at short gaps improves considerably and printing of a second NP becomes possible at any set gap (Figure 1D). Still, for gaps below $190 \mathrm{~nm}$ the experimental gap is larger than the set gap. The inset of Figure $1 \mathrm{~F}$ shows SEM images of the fabricated dimers at a set gap of $0 \mathrm{~nm}$, that is, aiming to connect the NPs. Even though a second particle was printed with a fairly well-controlled orientation, the average experimental gap was $151 \pm 12 \mathrm{~nm}$. These experiments show that lowering the temperature increase of the already printed NP by using a substrate with higher thermal conductivity leads to measurable improvements of the lateral resolution of optical printing. For the case of sapphire and Au NPs, there is still some residual photothermal repulsion that impedes the connection of NPs.

An alternative, complementary way of achieving better heat dissipation consists of increasing the contact area between the fixed NPs and the substrate. In order to test this approach, we evaluated the lateral resolution of optical printing colloidal Au $\mathrm{NP}$ next to $\mathrm{Au}$ nanodisks (NDs) fabricated by top-down methods onto sapphire substrates (Figure 2A; see SI.4 for details). NDs with a height of $50 \mathrm{~nm}$ and diameters of 70, 100, and $150 \mathrm{~nm}$ were used. The calculated temperature maps for the smallest and the largest NDs when set in the center of the printing beam are shown in Figure 2B,C, respectively. Even though the NDs have greater volume and equal or higher absorption cross sections than the spherical NPs (see SI.5), their temperature increase is around 5 times smaller. An example dark-field image of an array of $70 \mathrm{~nm}$ NDs is shown in Figure 2E, along with a SEM image of a ND and a typical 
scattering spectrum. The $60 \mathrm{~nm}$ Au NPs were printed close to the Au NDs in order to fabricate ND-NP dimers with different set gaps. Representative dark-field images of the obtained ND$\mathrm{NP}$ dimers are shown in Figure 2F for the $70 \mathrm{~nm}$ NDs. In addition, an example FE-SEM image of a ND-NP dimer fabricated with a set gap of $0 \mathrm{~nm}$ is shown with an attained gap of $35 \pm 5 \mathrm{~nm}$. Although particles look similar in FE-SEM images, the scattering spectrum reveals the presence of a ND and a spherical NP (Figure 2F).

Figure $2 \mathrm{D}$ shows the experimental gap versus the set gap for all disk sizes. Optical printing of NPs was possible for all set gaps and NDs sizes, achieving average gaps below $100 \mathrm{~nm}$, which were impossible to obtain with dimers of spherical $\mathrm{Au}$ NPs on glass or sapphire. This demonstrates that engineering the dissipation of heat via the NP geometry is another viable avenue for the improvement of lateral resolution of optical printing. Nevertheless, it was still not possible to connect the colloidal NPs to the NDs.

Figure 3 summarizes all results in a plot of the printing error versus the surface temperature increase of the fixed NP or ND.

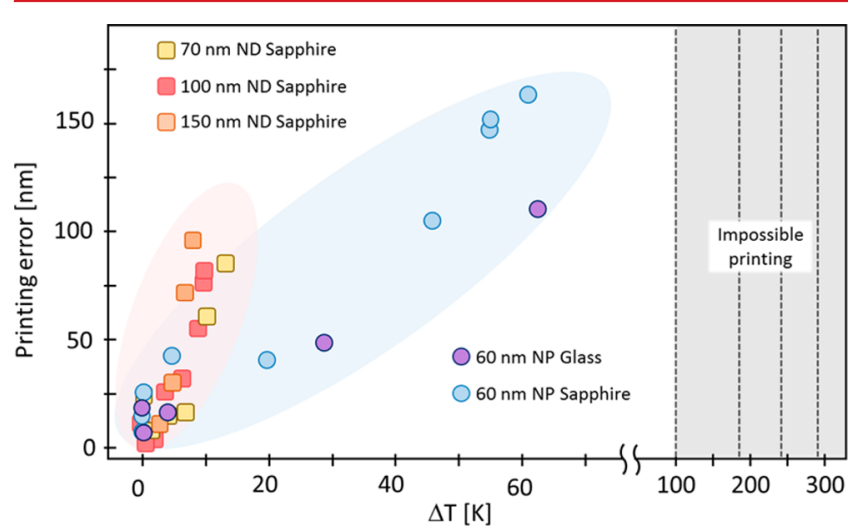

Figure 3. Absolute value of the average printing error versus the surface temperature increase of the previously fixed NP. The error was calculated as the difference between set gap and measured experimental gap. Vertical dotted lines in the gray area indicate experimental conditions where it was impossible to print.

The printing error is calculated as the difference between the set gap and the average experimental gap, that is, a measure of the deviation from the unperturbed behavior. The temperature increase $\Delta T$ of the fixed particle was computed for the particular conditions (absorption cross section, substrate, distance to the printing beam, and laser power) of each printing experiment (see SI.7). Remarkably, the results obtained with different particles, substrates, and set gaps group together in a common trend where the error increases monotonically with the surface temperature of the fixed particle. Furthermore, a more detailed inspection shows that the results obtained with fixed NPs and NDs group together while exhibiting slightly different trends, indicating that heat dissipation details, such as the problem geometry, are significant too. These results are in line with our previous report $^{24}$ and definitely confirm that the lateral resolution of optical printing is limited by light-induced heating. Interaction between NPs via scattered light (optical binding) ) $2,43-45$ may play only a minor role, as evident from the fact that the scattering cross sections at $532 \mathrm{~nm}$ of a $60 \mathrm{~nm}$ spherical NP and the $70 \mathrm{~nm}$ ND have similar magnitudes (see SI.5) but very distinct lateral resolutions of optical printing. Furthermore, the
$150 \mathrm{~nm} \mathrm{ND}$ has a scattering cross section 7-fold larger than a $60 \mathrm{~nm} \mathrm{Au} \mathrm{NP} \mathrm{but} \mathrm{presents} \mathrm{the} \mathrm{smallest} \mathrm{average} \mathrm{experimental}$ gap, that is, the weakest repulsions.

It is a noticeable fact that a relatively low surface temperature increase below $10^{\circ}$ can cause a repulsion comparable to the optical forces. In order to gain understanding, we analyzed all plausible mechanisms for this repulsion. First we note that the Reynolds number under the conditions of optical printing is considerably lower than 1 (see SI.8). Therefore, the liquid undergoes Stokes flows. In the following, we use the letter $U$ when referring to fluid velocity fields and $V$ for phoretic drift velocities of the particles.

A first possible origin of the thermally induced repulsion is natural convection. ${ }^{32}$ However, a straightforward analysis allows us to conclude that convective velocities could hardly exceed 1 $\mathrm{nm} / \mathrm{s}$ (see SI.8). Such fluid velocities translate into Stokes drag forces of $5 \times 10^{-7} \mathrm{pN}$, which are 6 orders of magnitude weaker than the optical counterpart. This means that the existent convective flows do not play any relevant role in the optical printing process. A second possibility is Marangoni convection. ${ }^{46}$ Recently, Ito et al. ${ }^{47}$ observed that if a bubble is formed around a hot NP, Marangoni convection up to 6 orders of magnitude stronger than natural convection can be driven by the surface tension of the water-air interface. The temperature threshold to form a bubble was reported to be around $600 \mathrm{~K}$ in several experiments of hot NPs ${ }^{48-51}$ under continuous wave excitation. Therefore, bubbles are not likely to be created by the low temperature increases during optical printing and Marangoni convection can be dismissed.

Having ruled out both natural and Marangoni convection, we propose a mechanism based on thermally induced flows that arise at a solid/liquid interface as a result of tangential temperature gradients. In the liquid boundary layer, a slip flow $U_{\mathrm{s}}$ is induced, which can be calculated as ${ }^{52,53}$

$$
\overline{U_{s}} \cdot \hat{t}=\chi \frac{\nabla T}{T} \cdot \hat{t}
$$

where $T$ is the temperature, $\hat{t}$ is the unit vector tangential to the surface, and $\chi$ is the thermo-osmotic coefficient or mechanocaloric cross-coefficient, ${ }^{53}$ a quantity related with the excess of specific enthalpy in the interfacial liquid layer compared to the bulk. Depending on the nature of the interaction (i.e., the sign of $\chi$ ) the fluid can be pushed toward the hotter or the colder region. If the surface is a fixed boundary, a long-range motion of the liquid is established known as thermo-osmotic flow. ${ }^{31} \mathrm{~A}$ microscale observation of such a flow was reported very recently by Bregulla ${ }^{53}$ et al. On the other hand, if the solid surface belongs to particle suspended in a nonisothermal liquid, the flow induces the migration of the particle, a phenomenon known as thermophoresis ${ }^{54-56}$ or the Soret effect. In the last context, usually an empirical description is employed, where the thermophoretic velocity $V_{\mathrm{tph}}$ of the particle is proportional to the local liquid temperature gradient $\nabla T$ and a thermodiffusion coefficient $D_{\mathrm{T}}$

$$
V_{\mathrm{tph}}=-D_{\mathrm{T}} \nabla T
$$

Because $D_{\mathrm{T}}$ is typically positive, ${ }^{57}$ particles move from hot to cold regions. It must be noted that despite being two different phenomena, the underlying physics of thermophoresis and thermo-osmosis are essentially the same with $D_{\mathrm{T}}$ and $\chi$ intrinsically related. ${ }^{52}$

The optical printing process involves temperature gradients on the liquid around the suspended NP being printed, as well 


\section{Thermophoresis}

A
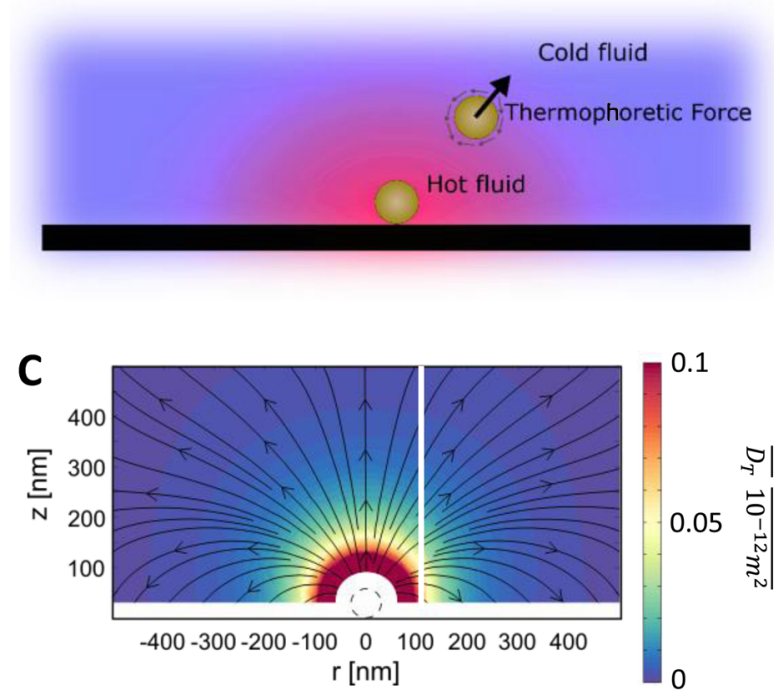

B
Thermo-osmosis
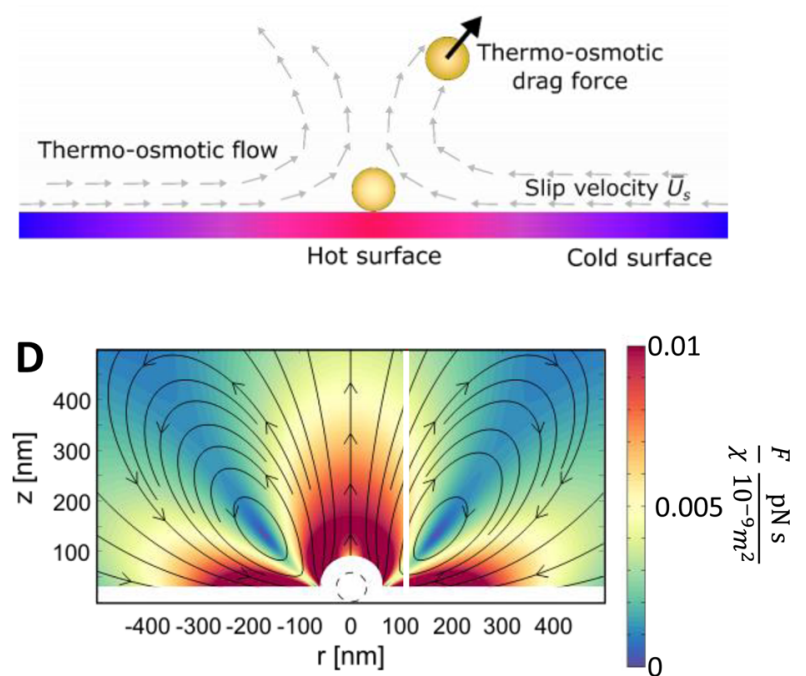

Figure 4. (A) Schematic of the thermophoretic force. A temperature gradient in the fluid pushes the NP away from the hot source. (B) Schematic of the thermo-osmotic force. A temperature gradient in the surface induces a slip velocity in the fluid. This creates a thermo-osmotic flow that exerts a drag force to the suspended NP. (C) Force map due to thermophoresis in units of the thermodiffusion coefficient $D_{\mathrm{T}}$ and (D) due to thermoosmotic flows in units of the thermo-osmotic coefficient $\chi$, acting on a $60 \mathrm{~nm} \mathrm{Au} \mathrm{NP}$ for positions around a fixed Au NP at $(0,0)$ on sapphire. Streamlines indicate the direction of the force and its magnitude is color coded. The center of the Gaussian laser focus is indicated with a vertical line. It has a waist of $266 \mathrm{~nm}$, wavelength of $532 \mathrm{~nm}$, and a power of $0.5 \mathrm{~mW}$, as in the experiments. White masked regions indicate unphysical regions within which the $60 \mathrm{~nm}$ printing particle would intersect the substrate or fixed particle.
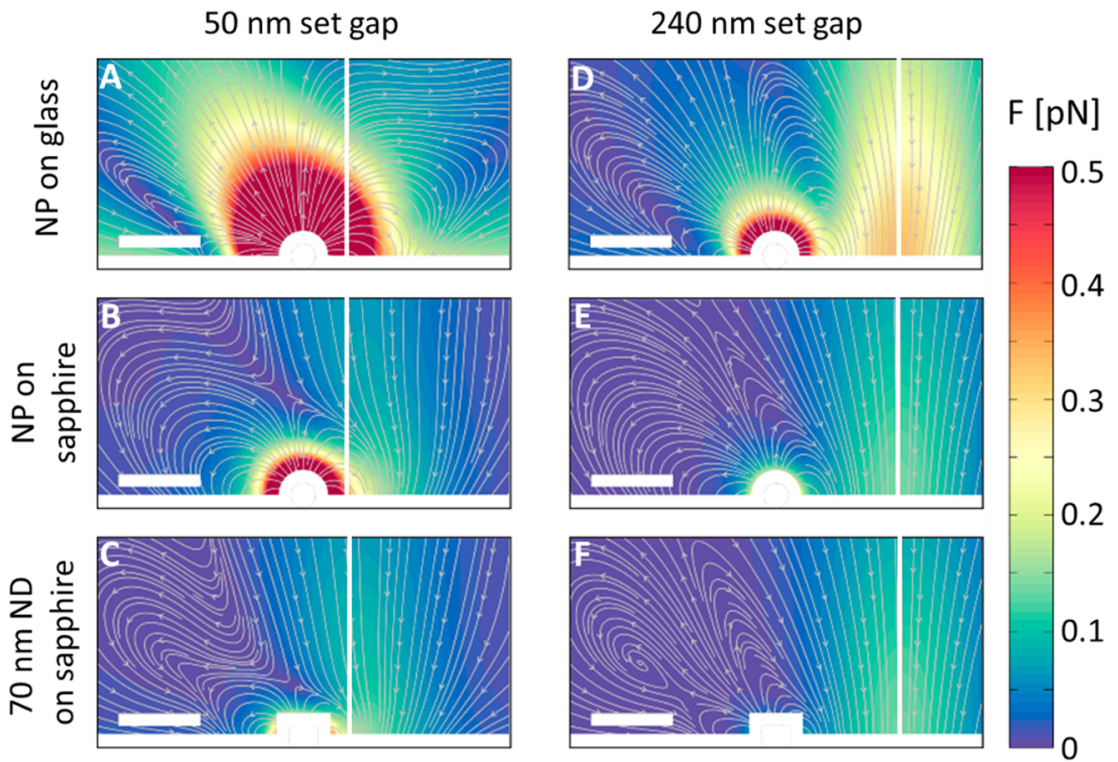

Figure 5. Maps of the net force (optical + thermophoresis + thermo-osmosis) acting on a $60 \mathrm{~nm} \mathrm{Au} \mathrm{NP} \mathrm{for} \mathrm{positions} \mathrm{around} \mathrm{a} \mathrm{fixed} \mathrm{Au}$ nanostructure at $(0,0)$. Panels A-C correspond to a $50 \mathrm{~nm}$ set gap of the beam for a $60 \mathrm{~nm}$ NP on glass, a $60 \mathrm{~nm}$ NP on sapphire and a $70 \mathrm{~nm}$ ND on sapphire, respectively. Panels D-F are the same configurations with a set gap of $240 \mathrm{~nm}$. Streamlines indicate the direction of the net force and its magnitude is color coded. The center of the Gaussian beam is indicated with a vertical white line. It has a waist of $266 \mathrm{~nm}$, wavelength of $532 \mathrm{~nm}$ and a power of $1.2 \mathrm{~mW}$ for glass and $0.5 \mathrm{~mW}$ for sapphire, as in the experiments. White-masked regions indicate unphysical regions within which the 60 $\mathrm{nm}$ printing particle would intersect the substrate or fixed particle. Scale bar: $200 \mathrm{~nm}$.

as on the fixed substrate (Figure 4A,B). Therefore, both thermophoresis and thermo-osmosis in principle could take place. In our previous report, ${ }^{24}$ the role of thermophoresis in optical printing was introduced but quantifying the magnitude of thermophoresis for metallic NPs remains in debate. ${ }^{54,58}$ Considering its high thermal conductivity, it has been argued that metallic NPs should not experience thermophoresis because the fluid in close vicinity of the NP is expected to have uniform temperature. ${ }^{53}$ However, this simplified picture could be wrong. It has been shown theoretically that the thermophoretic interaction is influenced by the properties of a finite layer of fluid around the NP surface, and that heat conductive NPs may present thermodifussion coefficients as high as dielectric NPs. ${ }^{59}$ In addition, two recent papers ${ }^{60,61}$ 
A

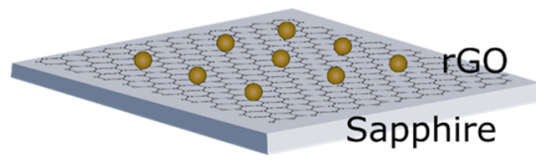

B

C

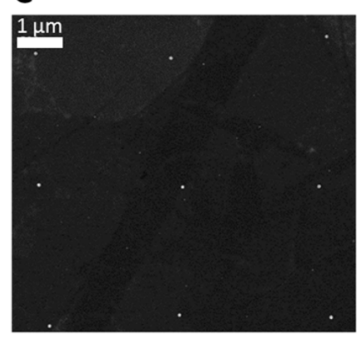

D

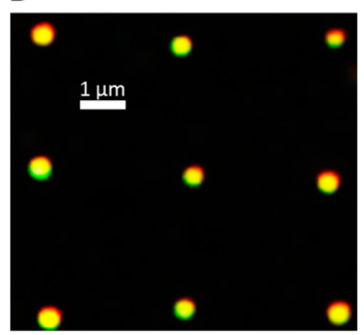

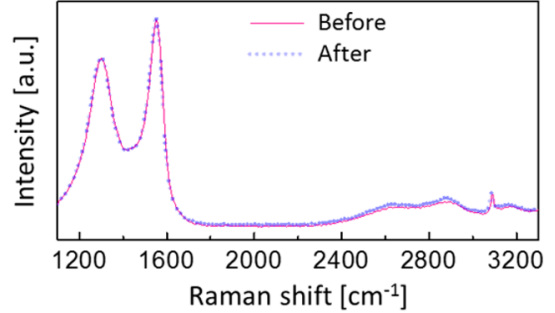

E

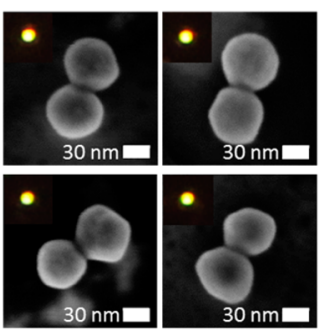

Figure 6. (A) Schematic of an optically printed grid of Au NPs on a sapphire substrate coated with reduced graphene oxide. (B) Raman spectrum of the rGO sample before (solid red) and after (solid blue) 2 min irradiation with the $532 \mathrm{~nm}$ beam at $1 \mathrm{~mW}$. The spectrum is unaltered, indicating that the sample is not being damaged by the beam. (C) FE-SEM and (D) dark-field images of $60 \mathrm{~nm}$ Au NPs optically printed on rGO. Panels C and D share the scale bar. (E) Au NP connected dimers on rGO fabricated by optical printing.

show experimentally and theoretically that thermophoresis is enhanced close to surfaces due to hydrodynamic boundary effects. This means that even if the thermodiffusion coefficient for metallic NPs in suspension was small, thermophoretic forces could still be appreciable close to surfaces. Here, we extend the discussion including the role of thermo-osmotic flows imposed by the substrate.

We simulated numerically the total force fields acting on a NP during the process of optical printing, considering the optical force, thermophoresis, and thermo-osmosis. Optical forces were calculated analytically following the approach described in ref 62 (further details in SI.6). The thermophoretic and thermos-osmotic forces were calculated based on temperature maps obtained from 3D finite element simulations (further details in SI.7). Thermophoretic velocities were obtained from the local temperature gradients using eq 2, and then transformed into corresponding drag forces using Stokes law (further details in SI.9). A typical example of the thermophoretic force field is shown in Figure 4C; the NP being printed tends to be repelled from the hot fixed NP on the substrate. Thermo-osmotic flows were obtained starting from the calculation of the slip velocity of a water layer at the solidliquid interface, according to eq 1 . Then, mass continuity requires the formation of convection-like fluid cells with closed streamlines. Once the water flow was obtained, a thermoosmotic drag force field was calculated according to Stokes law on a $60 \mathrm{~nm}$ NP. A typical force field due to thermo-osmosis is shown in Figure 4D.

Next, total force fields computing the optical, thermophoretic, and thermos-osmotic components can be calculated with only two free parameters: the unknown values of the thermodiffusion coefficient for a $60 \mathrm{~nm} \mathrm{Au} \mathrm{NP}\left(\left(D_{\mathrm{T}}^{\mathrm{Au}}\right)\right.$ and the thermo-osmotic coefficient for the substrate-water interface $\left(\chi^{\mathrm{p}-\mathrm{w}}\right)$. Because sapphire and glass substrates are equivalently functionalized with the same layer of polyelectrolyte, we consider both substrates to have the same value of $\chi^{\mathrm{p}-\mathrm{w}}$. Using an optimization algorithm (see SI.10 for details), we looked for the set of parameters $\left(D_{\mathrm{T}}^{\mathrm{Au}}, \chi^{\mathrm{p}-\mathrm{w}}\right)$ that best reproduced the average experimental gaps for all conditions explored. The obtained values turned out to be $D_{\mathrm{T}}^{\mathrm{Au}}=4.3 \times 10^{-12} \mathrm{~m}^{2} \mathrm{~s}^{-1} \mathrm{~K}^{-1}$ and $\chi^{\mathrm{p}-\mathrm{w}}=3 \times 10^{-9} \mathrm{~m}^{2} \mathrm{~s}^{-1}$. The estimated value of $\chi^{\mathrm{p}-\mathrm{w}}$ compares well to the only reported values in the literature, which are $\chi=0.2 \times 10^{-9} \mathrm{~m}^{2} \mathrm{~s}^{-1}$ for water on glass and $\chi=1.4$ $\times 10^{-9} \mathrm{~m}^{2} \mathrm{~s}^{-1}$ for water on glass functionalized with a block copolymer. $^{53}$

Figure 5 shows total force maps calculated using the optimized values of $\left(D_{\mathrm{T}}^{\mathrm{Au}}, \chi^{\mathrm{p}-\mathrm{w}}\right)$, for a NP being printed close to a $60 \mathrm{~nm} \mathrm{Au} \mathrm{NP}$ on glass and sapphire and close to a $70 \mathrm{~nm}$ ND on sapphire, at different set gaps. The center of the beam is depicted with a solid vertical line. Because the Reynolds number of the system is small (see SI.8), the NP motion is overdamped and force lines can be interpreted as trajectories. For a small set gap of $50 \mathrm{~nm}$ on glass (Figure 5A), the trajectories point away from the fixed NP and the target position; optical printing is impossible under these conditions. In contrast, for a fixed NP or ND on sapphire at the same small set gap (Figure 5B,C), there are trajectories capable of bringing the NP to the surface. However, these force lines lead to final positions further away from the fixed NP. For larger set gaps (Figure 5D-F) optical forces dominate and the trajectories lead to a final position according to the set-point.

The model presented so far quantitatively reproduces the experimental observations. Interestingly, for the optimum values of parameters $\left(D_{\mathrm{T}}^{\mathrm{Au}}, \chi^{\mathrm{p}-\mathrm{w}}\right)$, forces due to thermo-osmosis are considerable smaller than forces due to thermophoresis (see Sections SI.8 and SI.9). Furthermore, the parameter scan performed for optimization shows that the total force is practically independent of $\chi^{\mathrm{p}-\mathrm{w}}$. The prediction of our model using the optimum value $\chi^{\mathrm{p}-\mathrm{w}}=3 \times 10^{-9} \mathrm{~m}^{2} \mathrm{~s}^{-1}$ or $\chi^{\mathrm{p}-\mathrm{w}}=0$ is practically equivalent considering the experimental errors, meaning that we do not have evidence to affirm that thermoosmosis is a relevant phenomenon during optical printing.

A route to further reduce the photothermal heating is to employ substrates with even higher thermal conductivities, while keeping light absorption at the printing wavelength to a minimum. A suitable candidate is graphene. Its thermal conductivity has been claimed to be as has high as $5000 \mathrm{~W} /$ $\mathrm{mK}^{63}$ and the absorption of a single layer is only $2.3 \%$ at 532 
$\mathrm{nm} .{ }^{64}$ Thus, we tested the lateral resolution of optical printing on a sapphire substrate coated with one or two layers of reduced graphene oxide (rGO, Figure 6A, preparation details in SI.9). Before optical printing, the rGO presence was confirmed by Raman spectroscopy, using excitation light of $\lambda=532 \mathrm{~nm}$. Figure $6 \mathrm{~B}$ show a typical spectrum; the two main peaks observed at 1600 and $1330 \mathrm{~cm}^{-1}$ correspond to the $\mathrm{G}$ and $\mathrm{D}$ bands of rGO, respectively. The rGO layer is not damaged or altered by the irradiation levels used for optical printing, as demonstrated by a second Raman spectra acquired after 2 min of irradiation at $1 \mathrm{~mW}$. In this case, no extra surface modification of the substrate was necessary because no spontaneous attachment of NPs to the rGO was observed for at least $2 \mathrm{~h}$. The rGO substrate was not homogeneous. The laser power threshold for optical printing varied from region to region, revealing differences of surface charge density. We ascribe this to different degrees of reduction as a result of the rGO preparation procedure. Nevertheless, there were regions of the substrate where Au particles could be printed with a laser power of $0.5 \mathrm{~mW}$. Example FE-SEM and dark-field images are shown Figure $6 \mathrm{C}, \mathrm{D}$, respectively. In those regions, the possibility of connecting two Au NPs was tested by printing $\mathrm{Au} \mathrm{NP}$ dimers with a set gap of $0 \mathrm{~nm}$. Remarkably, in many cases connected dimers could be fabricated, as shown in Figure $6 \mathrm{E}$. A comparison to the previous experiments is not directly possible because the value of the thermos-osmotic coefficient $\chi$ for the rGO-water interface may be very different. Nevertheless, as it turned out from the quantitative analysis, the thermos-osmotic contribution is negligible in comparison to thermophoretic and optical forces. These results therefore indicate that the photothermal repulsion can be fully suppressed if heat dissipation is sufficiently efficient.

Conclusions. We have demonstrated that the lateral resolution of optical printing of colloidal NPs is limited by repulsive forces that arise from light absorption by the fixed NPs and consequent local heating. Increasing heat dissipation leads to significant resolution improvements. Using sapphire substrates, which have a 20 -fold higher thermal conductivity than glass, the minimum gap between optically printed $\mathrm{Au} 60$ $\mathrm{nm}$ NPs was reduced from 300 to $150 \mathrm{~nm}$. Heat dissipation can be increased also by improving thermal contact between the fixed NPs and the substrate. Even higher lateral resolution with gaps below $100 \mathrm{~nm}$ was achieved when printing Au NPs next to $\mathrm{Au}$ NDs on sapphire or glass. Spherical Au NPs could be connected directly by optical printing using an additional mono- or bilayer of reduced graphene oxide on sapphire substrates.

A detailed physical insight was obtained by comparing the experimental results to calculations, and evaluating all plausible photothermal phenomena as sources of the repulsive forces. Remarkably, numerical estimations of the temperature showed that small temperature increments below $10^{\circ}$ are enough to repel the optically driven AuNPs in the suspension. It was possible to conclude that neither natural nor Marangoni convection generate significant photothermal forces. Calculated force maps considering the optical, thermophoretic, and thermos-osmotic contributions were found to be fully consistent with the experimental observations. The only two free parameters, the thermodiffusion coefficient of the $60 \mathrm{~nm}$ $\mathrm{Au}$ NPs $D_{\mathrm{T}}^{\mathrm{Au}}$ and the thermo-osmotic coefficient for the interface between the polyelectrolyte-coated substrate and the water $\chi^{\mathrm{p}-\mathrm{w}}$, were obtained by scanning their values and contrasting the calculations to the experiments. The parameter scan showed that thermo-osmotic flows do not produce a considerable repulsion and that the dominant component arises from thermophoretic motion of the suspended NP. This is particularly interesting because currently it is under debate if metallic particles, which thermalize on femtosecond time scales, can be subject to thermophoresis. We found that the $60 \mathrm{~nm}$ AuNPs do experience considerable thermophoretic motion with a coefficient $D_{\mathrm{T}}^{\mathrm{Au}}=4.3 \times 10^{-12} \mathrm{~m}^{2} \mathrm{~s}^{-1} \mathrm{~K}^{-1}$. Because this is based on $3 \mathrm{D}$ calculations of the temperature maps, including the substrate, we believe this is the first reliable value reported for a metallic NP in suspension. We note that temperature fields around a hot nanostructure typically decay in lengths of about $100 \mathrm{~nm}$. Therefore, extreme temperature gradients as high as $10^{8}-10^{9} \mathrm{~K} / \mathrm{m}$ are produced. These conditions are significantly different from most theoretical and experimental reports, where small temperature gradients are considered. $^{36,37,65}$

The results of this work contribute to the general understanding of photothermal forces at the nanoscale and induced by high-temperature gradients and therefore their impact exceeds the field of optical printing. Reciprocally, it is envisioned that the technique can strongly benefit from a deeper comprehension of thermophoretic and thermo-osmotic phenomena of colloidal systems. For example, not only heat dissipation strategies but also surface modification strategies can be employed to reduce these effects. A reduction, or even sign reversal, of the coefficients $D_{\mathrm{T}}$ and $\chi$ can be pursued.

To sum up, we have improved significantly the lateral resolution of optical printing and at the same time gained detailed insight into photothermal forces at the nanoscale. As a result, the versatility of optical printing is expanded, broadening the range of systems based on colloidal NPs that can be fabricated with this technique. In addition, we have shown that optical printing can be combined with standard lithographic fabrication methods, allowing the incorporation of colloidal nanocomponents into previously patterned nanostructures with high accuracy. Finally, we have shown for the first time the combination of optical printing with $2 \mathrm{D}$ carbon materials. This is a novel route for the fabrication of template-free hybrid NPgraphene optoelectronic devices. These devices can merge the unique thermal and electrical conductivities of few-layered reduced graphene oxide films with the versatile photochemical properties of colloidal NPs with possible applications in biosensors, ${ }^{66-68}$ SERS, and light harvesting.

\section{ASSOCIATED CONTENT}

\section{Supporting Information}

The Supporting Information is available free of charge on the ACS Publications website at DOI: 10.1021/acs.nanolett.7b02713.

Details about the materials and methods (PDF)

\section{AUTHOR INFORMATION}

\section{Corresponding Author}

*E-mail: fernando.stefani@cibion.conicet.gov.ar.

ORCID

Julian Gargiulo: 0000-0002-4524-3423

Toshihiko Shibanuma: 0000-0002-2335-4958

Emiliano Cortés: 0000-0001-8248-4165

Fernando D. Stefani: 0000-0002-3277-7215 


\section{Funding}

This project was funded with the support of CONICET (PIP 112-201101-01035) and ANPCYT projects PICT-2010-2511, PICT 2013-0792, PICT 2014-3729, and PICT 2015-2285. EPSRC Reactive Plasmonics Programme (EP/522 M013812/ $1)$.

\section{Notes}

The authors declare no competing financial interest.

\section{ACKNOWLEDGMENTS}

P.A. acknowledges Programa "Viera y Clavijo" de la Agencia Canaria de Investigación, Innovación y Sociedad de la Información (ACIISI) y la Universidad de las Palmas de Gran Canaria (ULPGC). The Imperial College team acknowledges the EPSRC Reactive Plasmonics Programme (EP/ M013812/1) and the Lee Lucas Chair in Physics. F.D.S. also thanks the support of the Max Planck Society through a Partner Group grant. J.G. would like to thank Frank Cichos and Andreas Bregulla for discussion and to Maria Claudia Marchi for the FE-SEM imaging. We thank Alina Ghisolfi and Paloma Arroyo for helping with the design of the figures.

\section{REFERENCES}

(1) Sun, Y.; Xia, Y. Science 2002, 298, 2176-2179.

(2) Ye, X.; Jin, L.; Caglayan, H.; Chen, J.; Xing, G.; Zheng, C.; DoanNguyen, V.; Kang, Y.; Engheta, N.; Kagan, C. R.; Murray, C. B. ACS Nano 2012, 6, 2804-2817.

(3) Ghosh Chaudhuri, R; Paria, S. Chem. Rev. 2012, 112, 23732433.

(4) Xia, Y.; Xiong, Y.; Lim, B.; Skrabalak, S. E. Angew. Chem., Int. Ed. 2009, 48, 60-103.

(5) Sperling, R. A.; Parak, W. J. Philos. Trans. R. Soc., A 2010, 368, $1333-1383$.

(6) Tan, S. J.; Campolongo, M. J.; Luo, D.; Cheng, W. Nat. Nanotechnol. 2011, 6, 268-276.

(7) Flauraud, V.; Mastrangeli, M.; Bernasconi, G. D.; Butet, J.; Alexander, D. T. L.; Shahrabi, E.; Martin, O. J. F.; Brugger, J. Nat. Nanotechnol. 2016, 12, 73-80.

(8) Zhou, Y.; Zhou, X.; Park, D. J.; Torabi, K.; Brown, K. a; Jones, M. R.; Zhang, C.; Schatz, G. C.; Mirkin, C. a. Nano Lett. 2014, 14, 21572161.

(9) Wang, Y.; Wang, Y.; Breed, D. R.; Manoharan, V. N.; Feng, L.; Hollingsworth, A. D.; Weck, M.; Pine, D. J.; Figure, S.; Wang, Y.; Breed, D. R.; Manoharan, V. N.; Feng, L.; Hollingsworth, A. D.; Weck, M.; Pine, D. J. Nature 2012, 491, 51-55.

(10) Acuna, G. P.; Möller, F. M.; Holzmeister, P.; Beater, S.; Lalkens, B.; Tinnefeld, P. Science 2012, 338, 506-510.

(11) Vietz, C.; Kaminska, I.; Sanz Paz, M.; Tinnefeld, P.; Acuna, G. P. ACS Nano 2017, 11, 4969-4975.

(12) Fazio, B.; D’Andrea, C.; Foti, A.; Messina, E.; Irrera, A.; Donato, M. G.; Villari, V.; Micali, N.; Maragò, O. M.; Gucciardi, P. G. Sci. Rep. 2016, 6, 26952.

(13) Svedberg, F.; Li, Z.; Xu, H.; Käll, M. Nano Lett. 2006, 6, 26392641.

(14) Urban, A. S.; Lutich, A. A.; Stefani, F. D.; Feldmann, J. Nano Lett. 2010, 10, 4794-4798.

(15) Nedev, S.; Urban, A. S.; Lutich, A. a; Feldmann, J. Nano Lett. 2011, 11, 5066-5070.

(16) Do, J.; Fedoruk, M.; Jäckel, F.; Feldmann, J. Nano Lett. 2013, 13, 4164-4168.

(17) Violi, I. L.; Gargiulo, J.; Von Bilderling, C.; Cortes, E.; Stefani, F. D. Nano Lett. 2016, 16, 6529-6533.

(18) Spesyvtseva, S. E. S.; Dholakia, K. ACS Photonics 2016, 3, 719736.

(19) Guffey, M. J.; Miller, R. L.; Gray, S. K.; Scherer, N. F. Nano Lett. 2011, 11, 4058-4066.
(20) Huergo, M. A.; Maier, C. M.; Castez, M. F.; Vericat, C.; Nedev, S.; Salvarezza, R. C.; Urban, A. S.; Feldmann, J. ACS Nano 2016, 10, 3614-3621.

(21) Hoogenboom, J. P.; Vossen, D. L. J.; Faivre-Moskalenko, C.; Dogterom, M.; van Blaaderen, A. Appl. Phys. Lett. 2002, 80, 48284830.

(22) Guffey, M. J.; Scherer, N. F. Nano Lett. 2010, 10, 4302-4308.

(23) Bao, Y.; Yan, Z.; Scherer, N. F. J. Phys. Chem. C 2014, 118, 19315-19321.

(24) Gargiulo, J.; Cerrota, S.; Cortés, E.; Violi, I. L.; Stefani, F. D. Nano Lett. 2016, 16, 1224-1229.

(25) Wang, K.; Schonbrun, E.; Steinvurzel, P.; Crozier, K. B. Nat. Commun. 2011, 2, 469.

(26) Juan, M. L.; Righini, M.; Quidant, R. Nat. Photonics 2011, 5, $349-356$.

(27) Ohlinger, A.; Nedev, S.; Lutich, A. A.; Feldmann, J. Nano Lett. 2011, 11, 1770-1774.

(28) Cuche, A.; Canaguier-Durand, A.; Devaux, E.; Hutchison, J. A.; Genet, C.; Ebbesen, T. W. Nano Lett. 2013, 13, 4230-4235.

(29) Seol, Y.; Carpenter, A. E.; Perkins, T. T. Opt. Lett. 2006, 31, 2429-2431.

(30) Roxworthy, B. J.; Bhuiya, A. M.; Vanka, S. P.; Toussaint, K. C. Nat. Commun. 2014, 5, 1-8.

(31) Yang, M.; Ripoll, M. Soft Matter 2016, 12, 8564-8573.

(32) Donner, J. S.; Baffou, G.; McCloskey, D.; Quidant, R. ACS Nano 2011, 5, 5457-5462.

(33) Braun, M.; Cichos, F. ACS Nano 2013, 7, 11200-11208.

(34) Braun, M.; Bregulla, A. P.; Günther, K.; Mertig, M.; Cichos, F. Nano Lett. 2015, 15, 5499-5505.

(35) Lin, L.; Peng, X.; Wang, M.; Scarabelli, L.; Mao, Z.; Liz-Marzán, L. M.; Becker, M. F.; Zheng, Y. ACS Nano 2016, 10, 9659-9668.

(36) Duhr, S.; Braun, D. Proc. Natl. Acad. Sci. U. S. A. 2006, 103, 19678-19682.

(37) Reichl, M.; Herzog, M.; Götz, A.; Braun, D. Phys. Rev. Lett. 2014, 112, 198101.

(38) Jiang, H.-R.; Yoshinaga, N.; Sano, M. Phys. Rev. Lett. 2010, 105, 268302.

(39) Nedev, S.; Carretero-Palacios, S.; Kühler, P.; Lohmüller, T.; Urban, A. S.; Anderson, L. J. E.; Feldmann, J. ACS Photonics 2015, 2, 491-496.

(40) Simoncelli, S.; Summer, J.; Nedev, S.; Kühler, P.; Feldmann, J. Small 2016, 12, 2854-2858.

(41) Kyrsting, A.; Bendix, P. M.; Stamou, D. G.; Oddershede, L. B. Nano Lett. 2011, 11, 888-892.

(42) Dolinsky, Y.; Elperin, T. J. Appl. Phys. 2003, 93, 4321-4327.

(43) Chvátal, L.; Brzobohatý, O.; Zemánek, P. Opt. Rev. 2015, 22, 157-161.

(44) Dholakia, K.; Zemánek, P. Rev. Mod. Phys. 2010, 82, 17671791.

(45) Urban, A. S.; Carretero-Palacios, S.; Lutich, A. a; Lohmüller, T.; Feldmann, J.; Jäckel, F. Nanoscale 2014, 6, 4458-4474.

(46) Namura, K.; Nakajima, K.; Suzuki, M. Sci. Rep. 2017, 7, 45776.

(47) Setoura, K.; Ito, S.; Miyasaka, H. Nanoscale 2017, 9, 719-730.

(48) Baral, S.; Green, A. J.; Livshits, M. Y.; Govorov, A. O.; Richardson, H. H. ACS Nano 2014, 8, 1439-1448.

(49) Carlson, M. T.; Green, A. J.; Richardson, H. H. Nano Lett. 2012, 12, 1534-1537.

(50) Fang, Z.; Zhen, Y.; Neumann, O.; Polman, A.; García de Abajo, F. J.; Nordlander, P.; Halas, N. J. Nano Lett. 2013, 13, 1736-1742.

(51) Hou, L.; Yorulmaz, M.; Verhart, N. R.; Orrit, M. New J. Phys. 2015, 17, 013050.

(52) Anderson, J. Annu. Rev. Fluid Mech. 1989, 21, 61-99.

(53) Bregulla, A. P.; Würger, A.; Günther, K.; Mertig, M.; Cichos, F. Phys. Rev. Lett. 2016, 116, 188303.

(54) Würger, A. Rep. Prog. Phys. 2010, 73, 126601.

(55) Bickel, T.; Zecua, G.; Würger, A. Phys. Rev. E 2014, 89, 50303.

(56) Epstein, V. P. S. Eur. Phys. J. A 1929, 54, 537-563.

(57) Piazza, R.; Parola, A. J. Phys.: Condens. Matter 2008, 20, 153102.

(58) Michaelides, E. E. Int. J. Heat Mass Transfer 2015, 81, 179-187. 
(59) Giddings, J. C. C.; Shinudu, P. M.; Semenov, S. N. J. Colloid Interface Sci. 1995, 176, 454-458.

(60) Helden, L.; Eichhorn, R.; Bechinger, C. Soft Matter 2015, 11, $2379-2386$.

(61) Würger, A. Phys. Rev. Lett. 2016, 116, 138302.

(62) Agayan, R. R.; Gittes, F.; Kopelman, R.; Schmidt, C. F. Appl. Opt. 2002, 41, 2318-2327.

(63) Balandin, A. A.; Ghosh, S.; Bao, W.; Calizo, I.; Teweldebrhan, D.; Miao, F.; Lau, C. N. Nano Lett. 2008, 8, 902-907.

(64) Nair, R. R.; Blake, P.; Grigorenko, a. N.; Novoselov, K. S.; Booth, T. J.; Stauber, T.; Peres, N. M. R.; Geim, a. K. Science 2008, 320, 1308-1308.

(65) Jiang, H. R.; Wada, H.; Yoshinaga, N.; Sano, M. Phys. Rev. Lett. 2009, 102, 100-103.

(66) Cai, B.; Huang, L.; Zhang, H.; Sun, Z.; Zhang, Z.; Zhang, G. J. Biosens. Bioelectron. 2015, 74, 329-334.

(67) Artiles, M. S.; Rout, C. S.; Fisher, T. S. Adv. Drug Delivery Rev. 2011, 63, 1352-1360.

(68) Zhao, Y.; Zhu, Y. Nanoscale 2015, 7, 14561-14576. 